\title{
A LINEAR CONSTRUCTION FOR CERTAIN KERDOCK AND PREPARATA CODES
}

\author{
A. R. CALDERBANK, A. R. HAMMONS, JR., P. VIJAY KUMAR, N. J. A. SLOANE, AND \\ PATRICK SOLÉ
}

\begin{abstract}
The Nordstrom-Robinson, Kerdock, and (slightly modified) Preparata codes are shown to be linear over $\mathbb{Z}_{4}$, the integers mod 4 . The Kerdock and Preparata codes are duals over $\mathbb{Z}_{4}$, and the Nordstrom-Robinson code is self-dual. All these codes are just extended cyclic codes over $\mathbb{Z}_{4}$. This provides a simple definition for these codes and explains why their Hamming weight distributions are dual to each other. First- and second-order Reed-Muller codes are also linear codes over $\mathbb{Z}_{4}$, but Hamming codes in general are not, nor is the Golay code.
\end{abstract}

\section{INTRODUCTION}

Some of the best-known examples of nonlinear binary error-correcting codes that are better than any linear codes are the Nordstrom-Robinson, Kerdock, and Preparata codes [Ke72, NR67, Pr68, MS77]. Besides their excellent errorcorrecting capabilities, these codes are remarkable because the Kerdock and Preparata codes are "formal duals", in the sense that although these codes are nonlinear, the weight distribution of one is the MacWilliams transform of the weight distribution of the other [MS77, Chapter 15]. The main unsolved question concerning these codes has always been whether they are duals in some more algebraic sense. Many authors have investigated these codes and have found that (except for the Nordstrom-Robinson code) they are not unique and, indeed, that large numbers of codes exist with the same weight distributions [BLW83, Ca89, Ka82, Ka82a, Ka83, VL83]. Kantor [Ka83] declares that the "apparent relationship between these [families of codes] is merely a coincidence".

Although this may be true for many versions of these codes, we will show that, when properly defined, Kerdock and Preparata codes are linear over $\mathbb{Z}_{4}$ (the integers mod 4 ) and that as $\mathbb{Z}_{4}$-codes they are duals. All these codes are, in fact, just extended cyclic codes.

The version of the Kerdock code that we use is the standard one, while our version of the Preparata code differs from the standard one in that it is not a subcode of the Hamming code but of a nonlinear code with the same weight distribution as the Hamming code. Since the new construction is so simple, we propose that this is the "correct" way to define these codes.

Kerdock and Preparata codes exist for all lengths $n=4^{m} \geq 16$. At length 16 they coincide, giving the Nordstrom-Robinson code [NR67]. The $\mathbb{Z}_{4}$ version of the Nordstrom-Robinson code is the "octacode", a self-dual code of length 8

Received by the editors November 3, 1992 and, in revised form, December 7, 1992.

1991 Mathematics Subject Classification. Primary 94B05, 94B15, 94B60.

(c) 1993 American Mathematical Society $0273-0979 / 93 \$ 1.00+\$ .25$ per page 
over $\mathbb{Z}_{4}$ that is obtained when the Leech lattice is decomposed into eight copies of the face-centered cubic lattice. This result was announced in [FST93]. (The octacode itself is described in [CS92, CS93] and §3.)

The very good nonlinear codes of minimal distance 8 discovered by Goethals [Go74, Go76] and the high minimal distance codes of Delsarte and Goethals [DG75] also have a simple description as codes over $\mathbb{Z}_{4}$ (see [HKCSS]).

This work developed out of the discovery that four-valued sequences have excellent correlation properties [So89, Bo90, BHK92]) and was carried out independently by Hammons and Kumar [HK93] and (very slightly later) by the other three authors. Theorems 4-6 appear in Hammons's dissertation [Ha92]. In view of the considerable overlap we have now joined forces. This announcement is a compositum of our results, and full details will be given in [HKCSS].

For undefined terminology from coding theory see [MS77].

\section{CODES OVER $\mathbb{Z}_{4}$}

A quaternary linear code is an additive subgroup of $\mathbb{Z}_{4}^{n}$. Duality is defined with respect to the inner product $a \cdot b=a_{1} b_{1}+\cdots+a_{n} b_{n}(\bmod 4)$. We define three maps from $\mathbb{Z}_{4}$ to $\mathbb{Z}_{2}$ by

$\begin{array}{cccc}i & \alpha(i) & \beta(i) & \gamma(i) \\ 0 & 0 & 0 & 0 \\ 1 & 1 & 0 & 1 \\ 2 & 0 & 1 & 1 \\ 3 & 1 & 1 & 0\end{array}$

Then we construct binary codes from quaternary codes using the map $\phi: \mathbb{Z}_{4}^{n} \rightarrow$ $\mathbb{Z}_{2}^{2 n}$ given by

$$
\phi(a)=(\beta(a), \gamma(a)) .
$$

A binary code is $\mathbb{Z}_{4}$-linear if its coordinates can be permuted so that it is the image under this map of a linear code over $\mathbb{Z}_{4}$.

Theorem 1. (a) The binary image $\phi(D)$ of a quaternary linear code $D$ is linear if and only if

$$
a, b \in D \Rightarrow 2 \alpha(a) * \alpha(b) \in D,
$$

where * is componentwise multiplication.

(b) A binary linear code $C$ of even length is $\mathbb{Z}_{4}$-linear if and only if its coordinates can be permuted so that

$$
u, v \in C \Rightarrow(u+s(u)) *(v+s(v)) \in C,
$$

where $s$ is the "swap" map that interchanges the left and right halves of a vector.

Theorem 2. Binary Reed-Muller codes of length $n=2^{m} \geq 2$ and orders $0,1,2$, $m-1, m$ are $\mathbb{Z}_{4}$-linear.

Theorem 3. Extended Hamming codes of lengths $n=2^{m} \geq 32$ are not $\mathbb{Z}_{4^{-}}$ linear, nor is the Golay code of length 24.

Remark. In (3) if $u, v$ are represented by Boolean functions of degree $r$ and $(u+s(u)) *(v+s(v)) \neq 0$, then $(u+s(u)) *(v+s(v))$ is a Boolean function 
of degree $2 r-2$. So an $r$ th-order Reed-Muller code with $r \leq m / 2$ satisfies (3) provided $r \leq 2$ and we conjecture it does not satisfy (3) if $3 \leq r \leq m-2$. The first assertion of Theorem 3 establishes that $(m-2)$ nd-order Reed-Muller codes are not $\mathbb{Z}_{4}$-linear for $m \geq 5$.

Let $D$ be a quaternary linear code and $C=\phi(D)$ the corresponding binary code. In general $C$ is not linear, but we define the $\mathbb{Z}_{4}$-dual of $C$ to be $C^{\perp_{4}}=$ $\phi\left(D^{\perp}\right)$, where $D^{\perp}$ denotes the dual code to $D$, as in the following diagram.

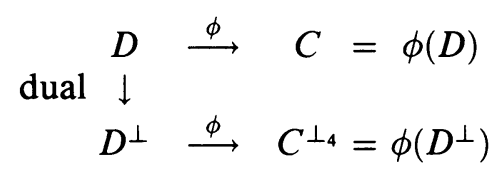

The familiar Hamming weight enumerator for a binary linear code $C$ will be denoted by $W_{C}(x, y)$. This weight enumerator is also well defined for binary nonlinear codes provided they are distance-invariant [MS77]. The symmetrized weight enumerator of a quaternary linear code $D$ is

$$
\operatorname{swe}_{D}(x, y, z)=\sum_{a \in D} x^{N_{0}(a)} y^{N_{1}(a)} z^{N_{2}(a)},
$$

where $N_{i}(a)$ is the number of components of $a$ congruent to $\pm i(\bmod 4)$. Then (see [CS93, K187])

$$
\operatorname{swe}_{D^{\perp}}(x, y, z)=\frac{1}{|D|} \operatorname{swe}_{D}(x+2 y+z, x-z, x-2 y+z) .
$$

Theorem 4. If $D$ is a quaternary linear code, then $C=\phi(D)$ and $C^{\perp_{4}}=\phi\left(D^{\perp}\right)$ are distance invariant, and

$$
\begin{gathered}
W_{C}(x, y)=\operatorname{swe}_{D}\left(x^{2}, x y, y^{2}\right), \\
W_{C^{\perp_{4}}}(x, y)=\frac{1}{|C|} W_{C}(x+y, x-y) .
\end{gathered}
$$

\section{Kerdock, Preparata and Nordstrom-Robinson codes}

Let $h_{2}(X) \in \mathbb{Z}_{2}[X]$ be a primitive irreducible polynomial of degree $m$. There is a unique monic polynomial $h(X) \in \mathbb{Z}_{4}[Z]$ of degree $m$ such that $h(X) \equiv h_{2}(X) \quad(\bmod 2)$ and $h(X)$ divides $X^{n}-1 \quad(\bmod 4)$, where $n=$ $2^{m}-1$ [So89, Ya90]. Let $g(X)$ be the reciprocal polynomial to

$$
\left(X^{n}-1\right) /((X-1) h(X)) \text {. }
$$

Theorem 5. The cyclic code generated by $g(X)$, extended by an overall parity check, is a quaternary code $D$ of length $2^{m}$ containing $4^{m+1}$ words. For $m$ odd $\geq 3$ the corresponding binary code $K=\phi(D)$ is the Kerdock code of length $2^{m+1}$ containing $2^{2 m+2}$ words and with minimal distance $2^{m}-2^{(m-1) / 2}$.

The proof is by showing that $D$ has a simple definition in terms of the relative trace function from $\mathbb{Z}_{4}[\xi]$ to $\mathbb{Z}_{4}$, where $\xi$ is a root of $h(X)$ (cf. [Bo90, BHK92]), and in this form it agrees with the definition of the Kerdock code given in [MS77, pp. 457-458]. 
Theorem 6. The cyclic code generated by $h(X)$, extended by an overall parity check, is a quaternary code $D^{\perp}$ dual to $D$. For $m$ odd $\geq 3$ the corresponding binary code $P=\phi\left(D^{\perp}\right)$ has length $2^{m+1}$, contains $2^{k}$ words, $k=2^{m+1}-2 m-$ 2 , has minimal distance 6 , and has the same weight enumerator as the Preparata code.

$P$ is the $\mathbb{Z}_{4}$-dual of $K$, both codes are distance invariant, and the weight distribution of one is the MacWilliams transform of the weight distribution of the other.

For example when $m=5$, we may take

$$
h(X)=\sum_{i=0}^{5} h_{i} X^{i}, \quad g(X)=\sum_{i=0}^{25} g_{i} X^{i},
$$

where $h_{0} \ldots$ and $g_{0} \ldots$ are 323001 and 11120122010303133013212213 . In this case the linear span of $P$ has minimal distance 2, which shows that $P$ is strictly different from Preparata's original construction [Pr68], for which the linear span is the extended Hamming code.

There is a distance-regular graph [BCN89] defined on cosets of our Preparata code which may be of some combinatorial interest.

In the case $m=3$, both $P$ and $K$ become the Nordstrom-Robinson code [NR67], and the quaternary code $D=D^{\perp}$ is the octacode. The latter may be defined as the extended cyclic code generated by $h(X)=X^{3}+3 X^{2}+2 X+3$ or as the unique quaternary self-dual code of length 8 which has the property that its binary image has minimal distance 6 [CS93] or as the "glue code" used to construct the Leech lattice from a direct sum of eight copies of the face-centered cubic lattice $A_{3}$ (note that $A_{3}^{\perp} / A_{3} \cong \mathbb{Z}_{4}$ ) [CS92, Chapter 24].

Theorem 7 [FST93]. The Nordstrom-Robinson code is the binary image of the octacode.

\section{REFERENCES}

[BLW83] R. D. Baker, J. H. van Lint, and R. M. Wilson, On the Preparata and Goethals codes, IEEE Trans. Inform. Theory 29 (1983), 342-345.

[Bo90] S. Boztaş, Near-optimal 4 $\phi$ (4-phase) sequences and optimal binary sequences for CDMA, Ph.D. dissertation, Univ. of Southern California, Los Angeles, 1990.

[BHK92] S. Boztaş, A. R. Hammons, Jr., and P. V. Kumar, 4-phase sequences with near-optimum correlation properties, IEEE Trans. Inform. Theory 38 (1992), 1101-1113.

[BCN89] A. E. Brouwer, A. M. Cohen, and A. Neumaier, Distance-regular graphs, SpringerVerlag, New York, 1989.

[Ca89] C. Carlet, $A$ simple description of Kerdock codes, Lecture Notes in Comput. Sci., vol. 388, Springer-Verlag, Berlin and New York, 1989, pp. 202-208.

[CS92] J. H. Conway and N. J. A. Sloane, Sphere-packings, lattices and groups, 2nd ed., Springer-Verlag, New York, 1992.

[CS93] Self-dual codes over the integers modulo 4, J. Combin. Theory Ser. A 62 (1993), 30-45.

[DG75] P. Delsarte and J. M. Goethals, Alternating bilinear forms over $G F(q)$, J. Combin. Theory Ser. A 19 (1975), 26-50.

[FST93] G. D. Forney, Jr., N. J. A. Sloane, and M. D. Trott, The Nordstrom-Robinson code is the binary image of the octacode, Proceedings DIMACS/IEEE Workshop on Coding and Quantization, DIMACS Series in Discrete Mathematics and Theoretical Computer Science, Amer. Math. Soc., Providence, RI (to appear). 
[Go74] J. M. Goethals, Two dual families of nonlinear binary codes, Electron. Lett. 10 (1974), 471-472.

[Go76] - Nonlinear codes defined by quadratic forms over $G F(2)$, Inform. Control 31 (1976), 43-74.

[Ha92] A. R. Hammons, Jr., On four-phase sequences with low correlation and their relation to Kerdock and Preparata codes, Ph.D. dissertation, Univ. of Southern California, November 1992.

[HK93] A. R. Hammons, Jr., and P. V. Kumar, On the apparent duality of Kerdock and Preparata codes, Abstracts, IEEE Internat. Sympos. Inform. Theory, San Antonio, TX, January 1993.

[HKCSS] A. R. Hammons, Jr., P. V. Kumar, A. R. Calderbank, N. J. A. Sloane, and P. Solé, The $\mathbb{Z}_{4}$-linearity of Kerdock, Preparata, Goethals and related codes, IEEE Trans. Inform. Theory, in press.

[Ka82] W. M. Kantor, An exponential number of generalized Kerdock codes, Inform. Control 53 (1982), 74-80.

[Ka82a] , Spreads, translation planes and Kerdock sets, SIAM J. Algebra Discrete Math. 3 (1982), 151-165, 308-318.

[Ka83] _ On the inequivalence of generalized Preparata codes, IEEE Trans. Inform. Theory 29 (1983), 345-348.

[Ke72] A. M. Kerdock, A class of low-rate nonlinear binary codes, Inform. Control 20 (1972), 182-187.

[K187] M. Klemm, Über die Identität von MacWilliams für die Gewichtsfunktion von Codes, Arch. Math. (Brno) 49 (1987), 400-406.

[VL83] J. H. van Lint, Kerdock and Preparata codes, Congr. Numer. 39 (1983), 25-41.

[MS77] F. J. MacWilliams and N. J. A. Sloane, The theory of error-correcting codes, NorthHolland, Amsterdam, 1977.

[NR67] A. W. Nordstrom and J. P. Robinson, An optimum nonlinear code, Inform. Control 11 (1967), 613-616.

[Pr68] F. P. Preparata, A class of optimum nonlinear double-error correcting codes, Inform. Control 13 (1968), 378-400.

[So89] P. Solé, A quaternary cyclic code, and a family of quadriphase sequences with low correlation properties, Lecture Notes in Comput. Sci., vol. 388, Springer-Verlag, New York and Berlin, 1989, pp. 193-201.

[Ya90] M. Yamada, Distance-regular digraphs of girth 4 over an extension ring of $Z / 4 Z$, Graphs Combin. 6 (1990), 381-394.

(A. R. Calderbank and N. J. A. Sloane) Mathematical Sciences Research Center, AT\&T Bell Laboratories, Murray Hill, New Jersey 07974

E-mail address, A. Calderbank: rc@research.att.com

E-mail address, N. Sloane: njas@research.att.com

(A. R. Hammons, Jr.) Hughes Aircraft Company, Canoga Park, California 91304

E-mail address: hammons@solar.usc.edu

(P. Vijay Kumar) Communication Science Institute, EE-Systems, University of Southern California, Los ANgeles, California 90089

E-mail address: kumar@lamarr.usc.edu

(Patrick Solé) Centre National de la Recherche Scientifique, Sophia - ANTipolis, 06560 VALBONNE, France

E-mail address: sole@mimosa.unice.fr 